\title{
Biparieto-occipital variant of Alzheimer's Dementia: Visual and praxis deficits
}

\author{
Keneilwe Malomo ${ }^{1}$ and Ontefetse Ntlholang ${ }^{2 *}$ \\ ${ }^{1}$ Registrar, Department of Medicine for the Elderly, Connolly Hospital, Blanchardstown, Dublin 15, Ireland \\ ${ }^{2}$ Consultant physician in Geriatric and Stroke Medicine, Department of Geriatric and Stroke Medicine, Beaumont Hospital, Dublin 9, Ireland
}

\begin{abstract}
We report a case of a 60 years old lady who presented with a two and half-year history of progressive cognitive decline affecting mainly visual perception and praxis skills. She used wrong words, had difficulty with pronunciation, substituting words and had expressive dysphasia. Her gait and hands tasks were dyspraxic. There was left-hand agraphesthesia, dyscalculia, intermittent left-right disorientation and dysdiadochokinesia and subtle left homonymous hemianopia. Her Magnetic Resonance Imaging of Brain showed global cerebral atrophy, most marked in the parieto-occipital areas bilaterally. Her symptoms, signs and brain imaging were typical of posterior cortical atrophy which is an atypical dementia which is often missed, and clinicians are not aware of. Awareness and recognition of posterior cortical atrophy may lead to early, accurate diagnosis and management.
\end{abstract}

\section{Introduction}

Posterior cortical atrophy (PCA) is a progressive neurodegenerative condition that affects parietal, occipital, and occipitotemporal regions of the brain. It was first described by Benson in 1988 and regarded as a variant of Alzheimer's disease in five patients [1]. The age of onset has been reported to be between 50 and 65 years [2]. Differential diagnoses include corticobasal degeneration [3], Creutzfeldt-Jakob disease [3] and dementia with Lewy bodies [3]. We report a case of PCA in a patient who presented with two and a half-year history of progressive cognitive decline affecting mainly visual perception and praxis skills.

\section{Case presentation}

A 60 years old right-handed lady was referred to a tertiary hospital with a two and a half-year history of change in cognitive function. She reported word finding difficulty, difficulty with expression and pronunciation of words. She also reported difficulties in going downstairs and non-specific visual problems. She saw an optometrist and was prescribed glasses that did not help. Furthermore, she reported that over the six months prior to presentation, her family told her she made mistakes with household tasks and had some difficulties operating the computer and writing at work. Her job description had changed from computer based to mail sorting.

Collateral history revealed that about two-and-a-half-years prior, she got lost in an airport she knows and missed flights. A year prior to presentation she started getting lost going to familiar relatives' houses and kept ringing for directions which were never the case before. Over the previous six months, the family noted that she made mistakes with household chores and also, they were informed of her difficulties at work, mainly operating computer and writing. Her condition gradually progressed since the family first noticed it to the extent that she was now misplacing things around the house, could forget details of the conversation and her mobility was also affected. She had gone much quieter and family attributed her symptoms to stress.
She has no personal or family history of neurodegenerative diseases and was not on medications. She is a non-smoker and drinks about 4 units of alcohol per week. She is educated to secondary school level and works as an office clerk.

On examination, she was casually dressed, had normal affect and mood was euthymic. She used wrong words, had difficulty with pronunciation, substituting words and had expressive dysphasia. Her gait and hands tasks were dyspraxic. She had left-hand agraphesthesia, dyscalculia and intermittent left-right disorientation and dysdiadochokinesia and subtle left homonymous hemianopia on examination.

Cambridge Cognitive Examination (CAMCOG), a part of the Cambridge Examination for Mental Disorders of the Elderly (CAMDEX) was 69/107 with the composite memory of 20/27. CAMCOG 20-1 score was 0 , serial 7 score 0 , WORLD 4, praxis 3, calculation 1 and visual construction 0 . Her delayed word recall (DWR) recall was 5/10 and recognition was 9/10. On Repeatable Battery for the Assessment of Neuropsychological Status (RBANS), there was evidence of dyscalculia, difficulty encoding and impaired drawing with one-sided clock drawing (Figure 1). Her immediate memory was 69 (1.8 percentile), visuospatial/construction 60 (0.4 percentile), language 68 (1.6 percentile), attention ungradable and delayed memory 78 (7.4 percentile). Magnetic Resonance Imaging (MRI) Brain showed global cerebral atrophy, most marked in the parieto-occipital areas bilaterally (Figure 2).

${ }^{\star}$ Correspondence to: Ontefetse Ntlholang, Department of Geriatric and Stroke Medicine, Beaumont Hospital, Dublin 9, Ireland, Tel: 0035318093000; E-mail: damaze2002@yahoo.com

Key words: posterior cerebral atrophy, Alzheimer's disease, cholinesterase inhibitors, biparieto-occipital atrophy, dementia

Received: June 22, 2018; Accepted: July 23, 2018; Published: July 30, 2018 

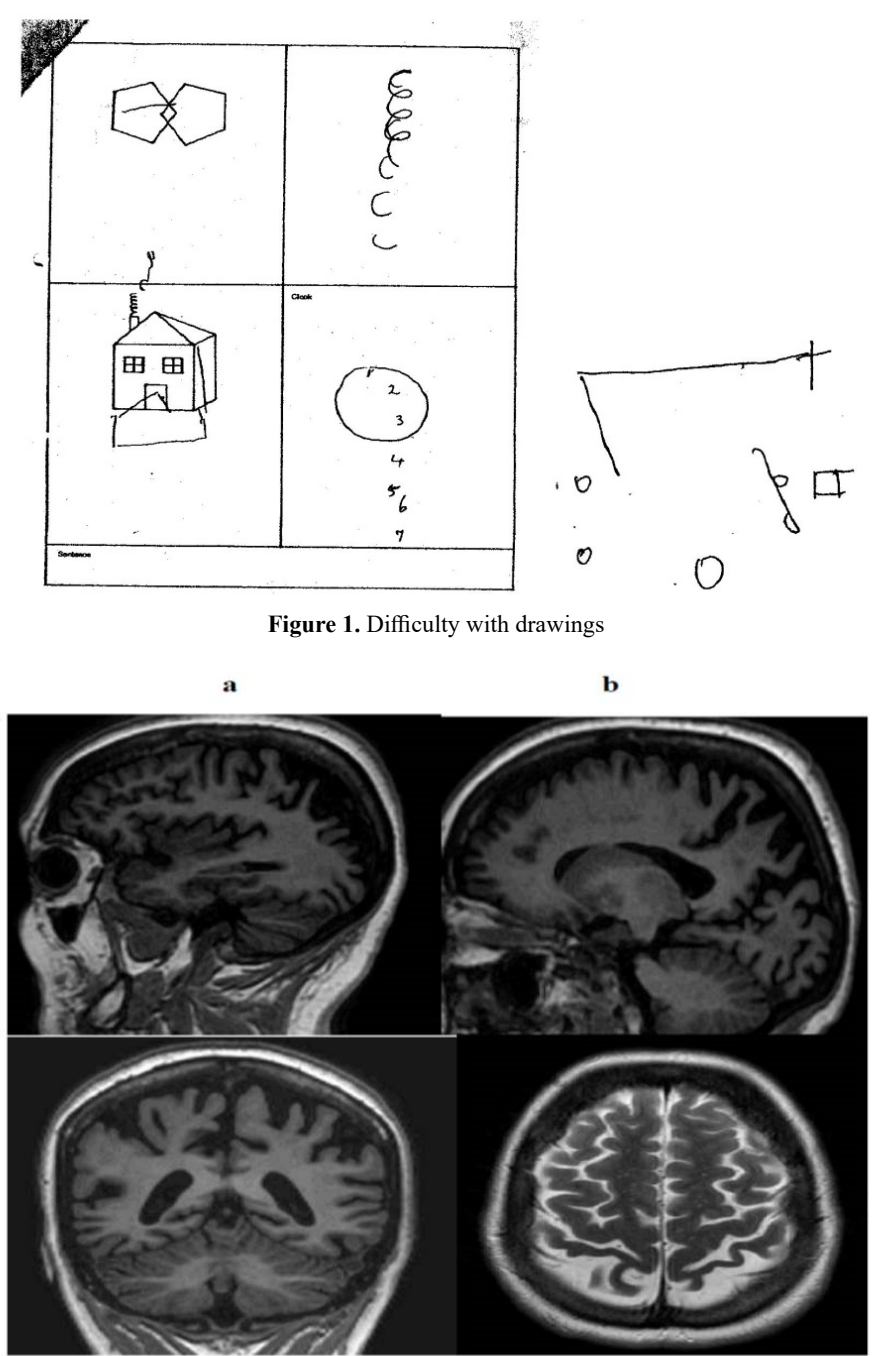

Figure 2. Magnetic Resonance Imaging (MRI) Brain revealing marked biparieto-occipital atrophy

A diagnosis of atypical Alzheimer's Dementia presenting as Posterior Cortical Atrophy was made. She was commenced on donepezil and seen by a multi-disciplinary team including the occupational therapist.

\section{Discussion}

PCA is still regarded as rare and most clinicians are not aware of it. The young age of onset and unusual symptoms might delay referral to

a specialist clinic and diagnosis [2]. Our patient had symptoms for two and a half years before being referred to a specialist. In the meantime, she was seen from eyes point of view and prescribed glasses even though her vision problem was not primarily from the eyes. The referral was mainly prompted by difficulties at work. Our patient's symptoms were attributed to stress and loss of confidence by her family that delayed presentation. Crutch et al. [2] reported that the presentation of PCA may lead to misdiagnosis of many patients as depressed, anxious or even malingering in the early stages of the disease.

Complete neurological examination in young people with a change in cognitive function whether affective disorders are suspected or not should be carried out in tandem with neuropsychological screening tests. In our case, occipital and parietal signs were present, and this raised suspicion before neuropsychological testing and neuroimaging. Previous studies have also elicited neurological signs depending on the area(s) of the brain involved [1,4,5]. Neuropsychological testing augments history and physical examination.

There was predominantly marked bilateral parieto-occipital atrophy on MRI brain in our case. The previous study reported parietooccipital atrophy $[4,5]$. Other imaging modalities include single photon emission computerized tomography (SPECT) [5], fluorodeoxyglucose positron emission tomography (FDG-PET) and Pittsburgh compound $\mathrm{B}$ (PIB)-PET [2]. Further investigations include cerebrospinal fluid examinations.

\section{Conclusion}

This case had long duration prior to presentation and had typical clinical and neuroimaging features of Posterior Cortical Atrophy which is an atypical dementia. Awareness and recognition of posterior cortical atrophy may lead to early, accurate diagnosis and management. Management includes a cholinesterase inhibitor [2]. Teaching and further researches are needed.

\section{References}

1. Benson DF, Davis RJ, Snyder BD (1988) Posterior cortical atrophy. Arch Neurol 45 789-793. [Crossref]

2. Crutch SJ, Lehmann M, Schott JM, Rabinovici GD, Rossor MN, et al. (2012) Posterior cortical atrophy. Lancet Neurol 11:170-178. [Crossref]

3. Renner JA, Burns JM, Hou CE, McKeel DW, Storandt M, et al. (2004) Progressive posterior cortical dysfunction: a clinicopathologic series. Neurology 63: 1175-1180. [Crossref]

4. Berthier ML, Leiguarda R, Starkstein SE, Sevlever G, Taratuto AL (1991) Alzheimer's disease in a patient with posterior cortical atrophy. J Neurol Neurosurg Psychiatry 54: 1110-1111. [Crossref]

5. Aharon-Peretz J, Israel O, Goldsher D, Peretz A (1999) Posterior cortical atrophy variants of Alzheimer's disease. Dement Geriatr Cogn 10(6):483-487.

Copyright: (C2018 Malomo K. This is an open-access article distributed under the terms of the Creative Commons Attribution License, which permits unrestricted use, distribution, and reproduction in any medium, provided the original author and source are credited. 